\title{
Stage IIA Uterine Sarcoma AJCC v7
}

National Cancer Institute

\section{Source}

National Cancer Institute. Stage IIA Uterine Sarcoma A/CC V7. NCI Thesaurus. Code

C115130.

Tumor involves adnexa. No regional lymph node or distant metastasis. 